\title{
Intensity Adaptive LSB Method Applying a Revised Matching
}

\author{
Javad Mohammadi Rad \\ Department of Computer Engineering \\ Eastern Mediterranean University (EMU) \\ Famagusta, Northern Cyprus (via Mersin 10 Turkey)
}

\begin{abstract}
This paper proposes a new steganographic algorithm in spatial domain. A filtering method selects some color components of pixelsin BMP cover imagesfor embedding according to their Most Significant Bits(MSB) value. The components whose MSB value isgreater than a particular threshold,are qualified and the other ones will be skipped. This mechanism makes the retrieval of embedded message possible. Moreover, a matching technique ensures the most possible closeness of new intensity generated in embedding process to its original value.Performance of proposed method is evaluated by some measures namely MAE, MSE,LMSE,L ${ }^{\mathrm{P}}$-Norm, SNR, PSNR, and NCC according to which, proposed method offers up to $40 \%$ better results in some measures compared to two other methods; LSB and SLSB.
\end{abstract}

\section{Keywords}

Filtering, Intensity Adaptive LSB (IALSB), LSB Matching, LSB Plane, LSBSteganography,MSB, Performance Measure, SLSB, Spatial Domain, Threshold.

\section{INTRODUCTION}

In the age of computers and communications, data transmission through internet has made it possible to have afast access to data all over the world.In order to be able totransmit data securely against eavesdroppers, some techniques have been developed. Steganography is one of these techniques.

The word "Steganography" is originally derived from the Greek words steganos (covered) and graphy (writing) and literally means covered writing[1]. Using steganography, information is embedded in a medium such as image, audio, video, or text file called carrier in a way that it is not detectable by others[2]. Digital images are the most popular ones due to their variety and frequencyon the Internet. In image steganography secret information is embedded exclusively in images [3].The input image in which a secret message will be embedded is called cover image and the image containing the secret message is known as stego image[4]. Another technique used in secret communications is Cryptography in which secret information is transformed to non-understandable form, but in steganography, no one knows about the existence of secret information transmitting between sender and receiver. Thus, steganographic algorithms provide more security in comparison to cryptographic approaches[5].

Oneof the major types applied in steganography is spatial domain. Spatial domain methods also known as image domain methods, substitution methods, or noise insertion methods,embed the secret message in the intensity of the cover image pixels. They work at level of LSBs. Firstup to the fourth LSB of the pixelmay besubstituted in the pixel with the bits of secret data by steganographer.Since only insignificant modifications are exerted in the embedding process, it is assumed that these changes will not be detected by an attacker.
There is a trade-off between the embedding capacity (payload) and cover image distortion. Replacing more LSBs would cause more stego image distortion. Spatial domain methods benefit from simplicity andhigh payload capacity, but they are vulnerable against noise, lossy compression, image filters, cropping, and rotation. They also affect statistical properties of the image. BMP and GIF are the image formats that spatial domain methods work on[2],[5],[6], and [7].In this paper, the LSB and SLSB methodswill be reviewed and then a new steganographic technique and its two approaches will be introduced, investigated, and evaluated.

\section{REVIEW OF RELATED WORKS}

Two of fundamental inspiring steganographic methods to the new proposed method are as follows:

\subsection{Least Significant Bit (LSB) Method}

This technique is an instance of spatial domain methods. It is a simple, common approach whichplaces the bits of secret data at the LSB of the pixel in the cover image. Using a 24-bit BMP cover image, the LSB of red, green and blue (RGB) components participate in embedding process, since each of them is represented as a byte. In other words, it can store 3 bits of secret dataperpixel. While,for a GIF or 8-bit BMP file this value is equal to 1 bit per pixel. On average, around half of the LSBs in a cover image are needed to be modifiedto embed a secret message using the maximum cover size.

The distribution of embedding pixelsthrough the cover imagecan be contiguous or scattered. In the first approachalso known as sequential embedding, bits of secret message are embedded in the cover pixelsconsecutively. The location of first embedding pixel must be known for extraction of secret message. While,the second approachalsocalledrandom interval embedding uses a pseudorandom number generator (PRNG) to spread the secret data over the cover image in a rather random manner. Using a stego-key as a seed for a PRNG, a random sequence of indices is generated to determine the distance between two embedded bits pseudorandomly. The receiver can reconstruct the random sequence and extract the secret message byhaving access to the seedand knowledge of the PRNG [5],[7],[8],[9],[10].

Following [11], in this paper, in order to visualize the distributionof replacements and embedding colors and pixels in stego image,LSB plane is applied.Referring to the LSB plane and its guide, the modified pixels and components of the cover image in the stego image can be found correspondingly.

The LSB plane of sequential LSB method on the Pepper.bmp cover image with the secret message mentioned in section 4andreplacing $3 \mathrm{bit} / \mathrm{pixel}$ is as follows: 


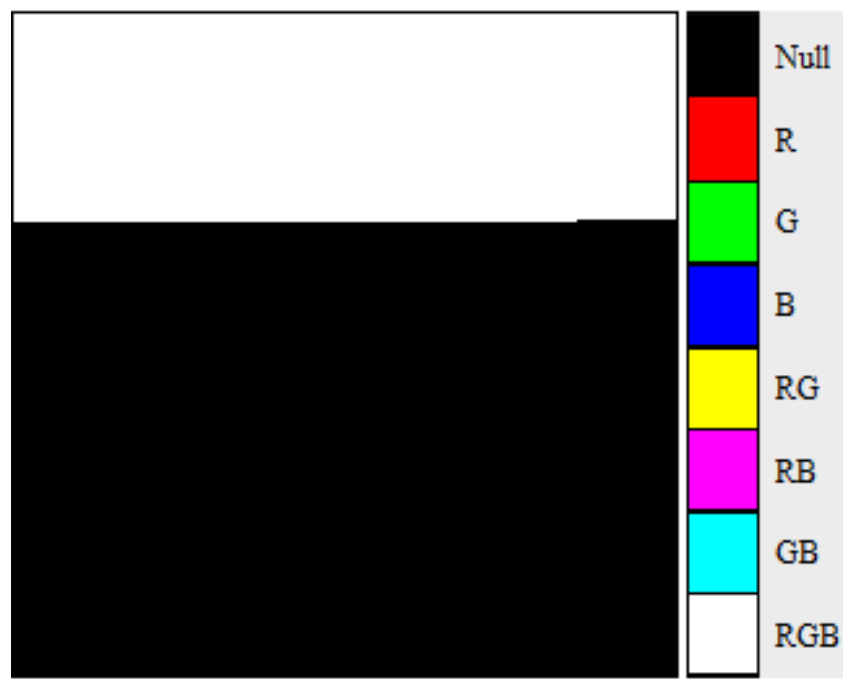

Figure 1.LSB plane of sequential LSB method

As shown in the Figure 1, sequential LSB method starts from the beginning and embeds the secret bits in all components of the pixels consecutively until the whole message is covered.

\subsection{Selected Least Significant Bit (SLSB) Method}

Itbelongs to spatial domain group[12].Many of spatial domain steganographic algorithms embed secret information in the LSB of all red, green, and blue(RGB) colors of the 24-bit cover pixels that may lead to major distortions. These distortions may not be detectable by human eye, but they will be detected by some statistical analyses such as RS[13] and Sample Pairs[14] As a solution, SLSB algorithm selects one color out of three (RGB) applying a preliminary Sample Pairs analysis[15] by which a color that offersmore diversity and consequently less considerable changes is selected [12], [15].

After color selection, SLSB algorithmfilters the cover image (evaluatively) using an edge detection filter such as Laplace, Prewitt, Roberts, Sobel, or more likely Roque filter[12],[16],[17] and embeds the secret data in the areas that achieve a better ratein filtering process. The filter employs most significant bits of the pixels andleaves less significantones for embedding.This ensures that the embedded information be retrievable. That is to say, in extracting process, reapplication of the filter will choose the same pixels and components selectedfor the concealing process[12].

According to[12] andfollowing[18] and [19],in order to havethe final color closest to the original color, LSB Matching techniquecalculates the distance between the steganographic and original color. If it is greater than a certain threshold determined by the number of bits to hide, the color is decremented to get a final color closest to its origin.

Applying a histogram analysis to the SLSB method's stego image reveals that there are only changes in the histograms of illumination and the color selected by the algorithm as the optimal color of embedment[12].

The LSB plane of SLSB method on the Pepper.bmp cover image with the secret message mentioned in section 4 and replacing 3 bit/pixel ( 3 bits of selected color) is as follows:

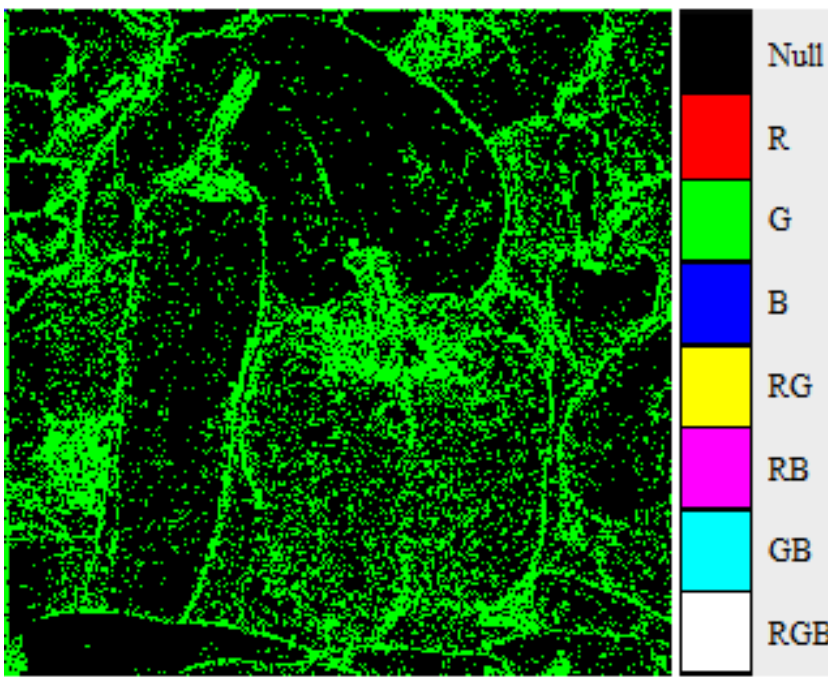

Figure 2.LSB plane of SLSB

According to the Figure 2, SLSB algorithm has selected the green color of pixels emphasizing on the edges for embedding.

\section{NEW PROPOSED METHOD; INTENSITY ADAPTIVE LEAST SIGNIFICANT BIT (IALSB)}

\subsection{Description}

Proposed method; IALSB is an instance of spatial domain group.Following [12], this method applies an evaluative highintensity filter to the components of the cover pixels and embeds the secretinformation in the areas that are qualified according to the filter. That is to say,the secret information is embedded in the LSB of the components whose MSB value aregreater than a particular threshold. This mechanism ensures the retrievability of embedded information. After embedment, a matching technique is appliedto moderate the new generated color toward its origin. The first bit adjacent to the least significant bit or bits would change in favor of closing new generated color to its originand lead to less visibility of stego image distortions. According to the description of new proposed method, the components (bytes) of the cover pixels are structured as follows:

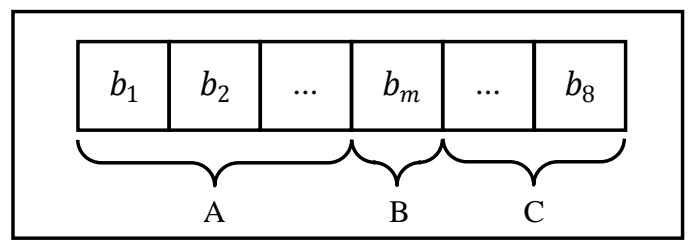

Figure 3. Color structure in IALSB

Section A of the Figure 3 is most significant bits section which will be evaluated in the filtering step. This part will never be modified to ensure the secret information retrieval. Section B of the Figure 3is the matching bit which may be modified to moderate the distortions generated in embedding step. The last part; Section $\mathrm{C}$ of the Figure 3 includes least significant bit or bits that carry the secret information. The certain indices of MSB and matching sections (A and $B$ respectively) are dependent to the number of the bits that section $\mathrm{C}$ employs. Applying more LSBs for embedding will push the sections A and $\mathrm{B}$ to the left part of the color. For example, considering $\mathrm{b}_{7}$ and $b_{8}$ as section $C$, the section $B$ includes $b_{6}$ and consequently section A consists of $b_{1}, b_{2}, b_{3}, b_{4}$, and $b_{5}$. The flow of embedding process is as Figure 4 shows. 


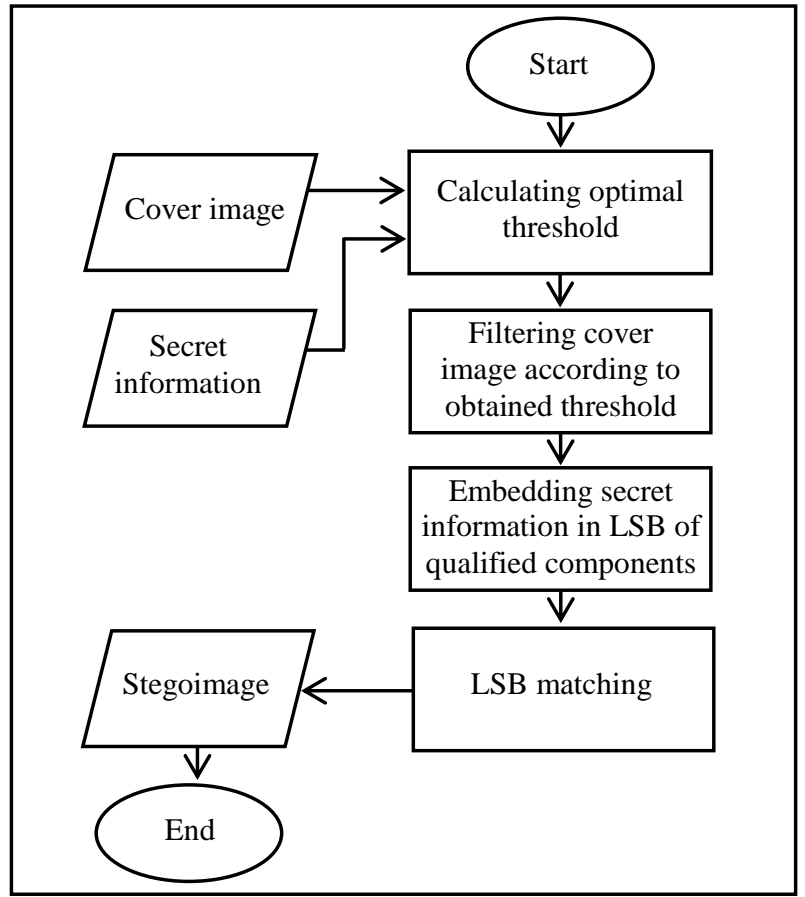

Figure 4. The flow of embedding process

\subsection{Calculating a Particular Threshold}

Proposed method applies a particular threshold to determine the pixels and components participate in embedding process. The components whose MSB valueisgreater than the threshold are qualified for embedding the secret information.According to [20], assuming $k$ as the number of color components needed to embed secret message:

$$
k=\left\lceil\frac{l}{n}\right\rceil
$$

wherel is the length of the secret bit stream (secret message in bits) and $n$ is number of LSB used per component for embedding. To obtain the optimal thresholdof $m$ covercomponents, the MSB value of all mcomponents should be sorted in descending order. According to the equation (1), the $k^{\text {th }}$ element of the sorted MSBs refers to the optimal threshold. The illustration is as follows:

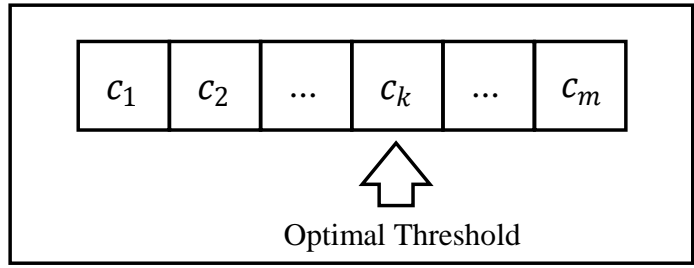

Figure 5.Optimal threshold determination $\left(c_{i} \geq c_{i+1}\right)$

The figure 5 illustrates the determination of optimal threshold; $c_{k}$ from the sorted MSBs $\left(c_{i} \geq c_{i+1}\right)$. This is the maximal possible threshold which ensures embedding of the secret message with the most scatter through the cover image. Applying a threshold greater than $c_{k}$ will cause whole the secret message not be embedded fully, and using a threshold smaller than $c_{k}$ will cause the secret message not be scattered mostly through the cover image.

\subsection{LSB Matching}

According to [21], in the LSB replacement, if the secret bit does not match the cover LSB, then LSB matching adds +1 or -1 is to the correspondingpixel value randomly. Statistically, generated asymmetry artifacts can be avoided.

In this work, LSB matching has been adapted for any arbitrary number of LSB. As illustrated in the Figure 3, a matching bit $\left(b_{m}\right)$ is considered for each component of the cover pixels adjacent to LSB section. This bit may be changed in order to moderate the distortion generated by embedment of secret message. Following[12], [18], [19], and [21], the $b_{m}$ will be valued as follows:

$$
b_{m}=\left\{\begin{array}{lr}
1 & \left|c-c^{\prime}\right|>\left|c-c^{\prime \prime}\right| \\
0 & \left|c-c^{\prime}\right|<\left|c-c^{\prime \prime}\right| \\
1 \text { or } 0 & \text { Otherwise }
\end{array}\right.
$$

where $\mathrm{c}$ is the original value of the cover component, $c^{\prime}$ is the stego value of the component considering $b_{m}$ as zero, and c" is stego value of the component considering $b_{m}$ as one. As equation (2) expresses, matching $\operatorname{bit}\left(b_{m}\right)$ of each component will be set to a valuewhich minimizes the difference between the original and stego value of that color component.

\subsection{Two Approaches for Proposed Method}

The proposed method can be applied in two approaches. First approach is employing all colorsof the cover pixels for embedding. It benefits from high payload capacity, but it may cause some distortions due to modification of all components in some cover pixels. This approach can be named Multi-color IALSB.

Applying Multi-color IALSB, in $\mathrm{aM} \times \mathrm{N}$ pixel 24-bit cover image, the optimal threshold is the $k^{\text {th }}(k$ from (1)) element of 3MNdescending sorted MSBs of all cover components.

The LSB plane of Multi-color IALSB approach on the Pepper.bmp cover image with the secret message mentioned in section 4 and replacing 3 bit/pixel (if all 3 components are qualified) is as follows:

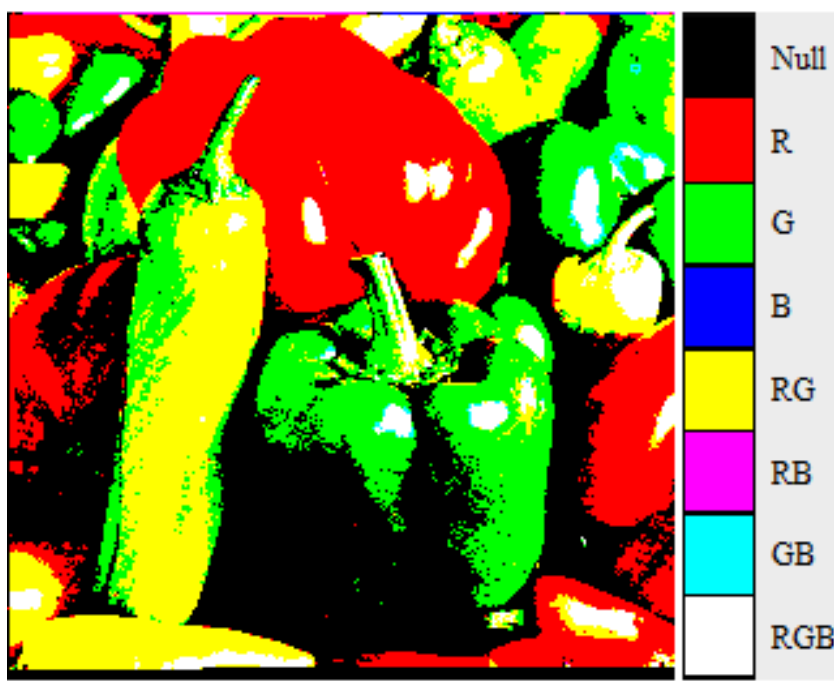

Figure 6.LSB plane of Multi-color IALSB

According to the Figure 6, the secret message is embedded in various components of the pixels. In some pixels, individual red or green components are used for embedding. In some others, combinations of components are employed. Referring to the LSB plane guide, yellow pixels state that only the red and green components of corresponding pixels have MSB values greater than the applied threshold and due to this contain secret information, but their blue components contain no embedment. White pixels of the LSB plane state that corresponding pixels in stego image contain embedment in their all red, green, and blue components, because bright pixels contains high intensity of RGB. On the other hand, in the black pixels none of components are qualified to be employed in the embedment. 
Second approach is employing one of the colorsout of three which consequently leads to lower distortion and payload.This approach can be called Single-color IALSB [12].

The target color in Single-color IALSB will bethe one which has the most qualified components applying the corresponding local optimal threshold.According to [12] and [15], more frequency of qualified components leads to less considerable changes.Applying Single-color IALSB, the local optimal threshold for one colorin a $\mathrm{M} \times \mathrm{N}$ pixel 24-bit cover image is obtained by selecting the $k^{\text {th }}$ ( $k$ from (1)) element ofMN descending sorted MSBs ofthe same color.

After color selection, all remaining steps of embedding are the exerted to the components of selected color.

The LSB plane of Single-color IALSB approach on the Pepper.bmp cover image with the secret message mentioned in section 4 and replacing 3 bit/pixel ( 3 bits of selected color) is as follows:

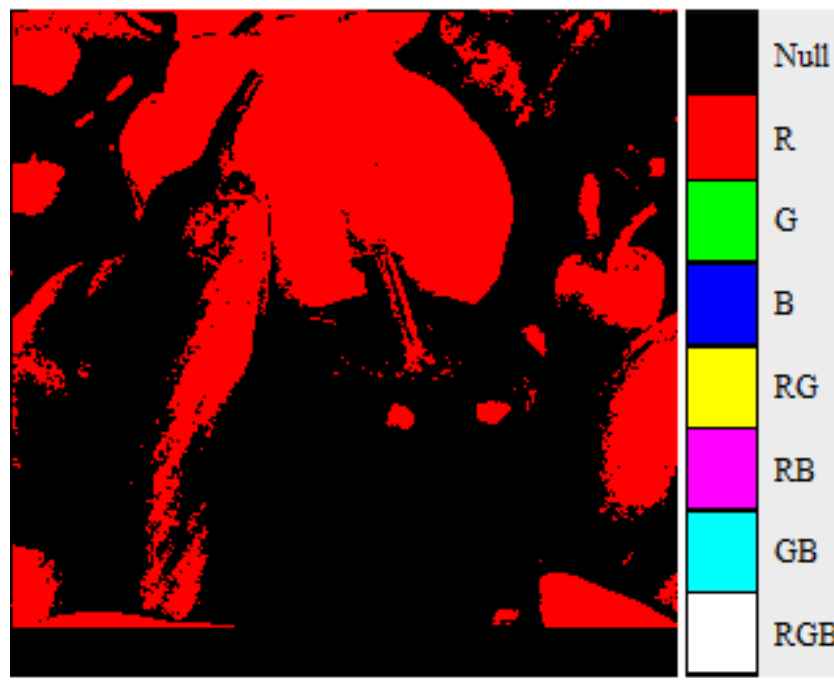

Figure 7.LSB plane of Single-color IALSBapproach

The figure 7 expresses that the red color of the cover pixels is the one which has the most frequent qualified components. The secret message is embedded in the high intensity red components whose MSB values greater than the corresponding threshold.

\section{EXTRACTION PROCESS}

Extraction process of the secret information is started with calculating the threshold used in embedding process. Using stego key including $l$ and $n$ (from (1)), $k$ is obtainable[20]. The threshold will be the $k^{\text {th }}$ element of the stego image MSBssorted in descending order knowing that the stego MSBs are not changedin the embedding process. Having the stego image, the stego key ( $l$ and $n$ from (1)), and the obtained threshold, the secret information can be extracted by filtering the stego image. In Single-color IALSB extraction process, all steps are performed by using local optimal threshold of the selected color.

Then (from (1)) LSB of qualified components in filtering process make the secret bit stream consecutively. The flow of extraction process is shown in Figure 8.

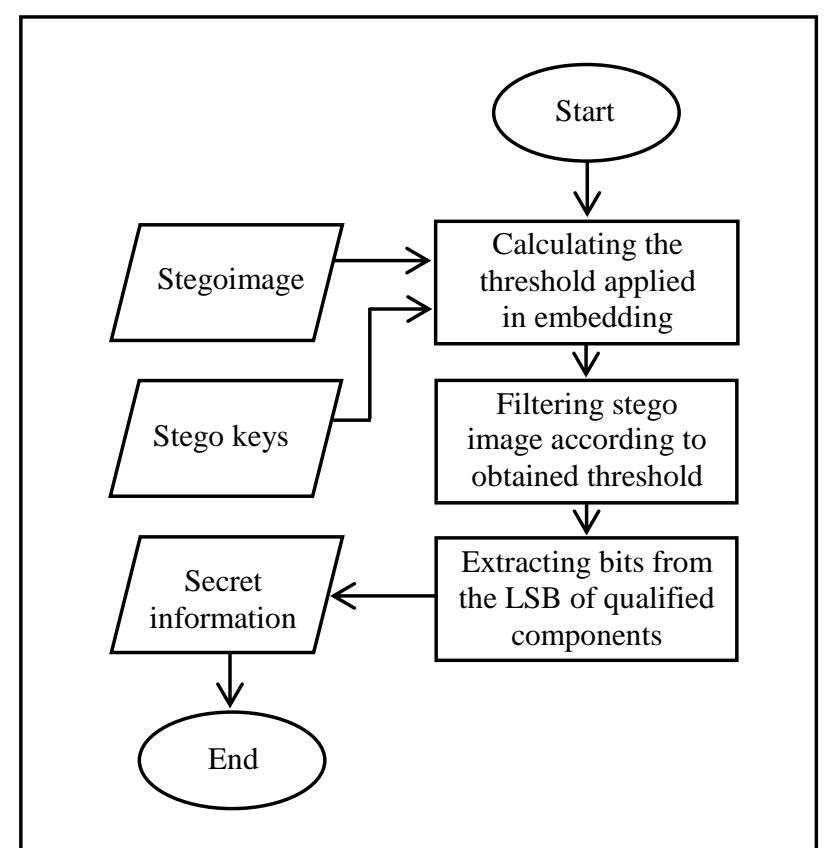

Figure 8. The flow of extraction process

\section{EXPERIMENTAL SETUP}

To evaluate the new proposed approaches, twenty frequentlyused 512×512 24-bitBMP cover images, whose sizes are 786,486 bytes namely Baboon.bmp, Barbara.bmp, Boats.bmp, Cove.bmp, Earth.bmp, F16.bmp, GoldHill.bmp, House.bmp, Lena.bmp, Monarch.bmp, Oakland.bmp, Peppers.bmp, Sailboat.bmp, San-Diego.bmp, Splash.bmp, Tiffany.bmp, Tulips.bmp, Woodland- Hills.bmp, Yacht.bmp, and Zelda.bmp are selectedwhose thumbnails are as follows respectively:
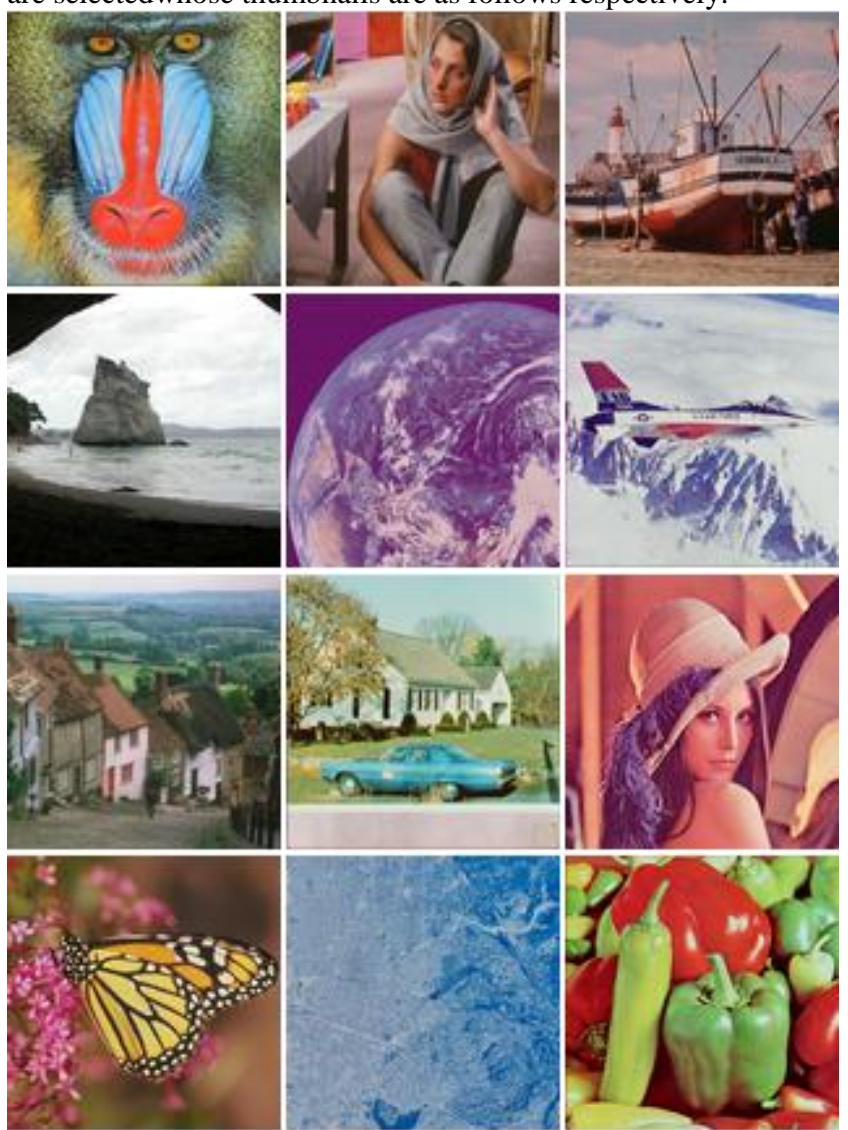


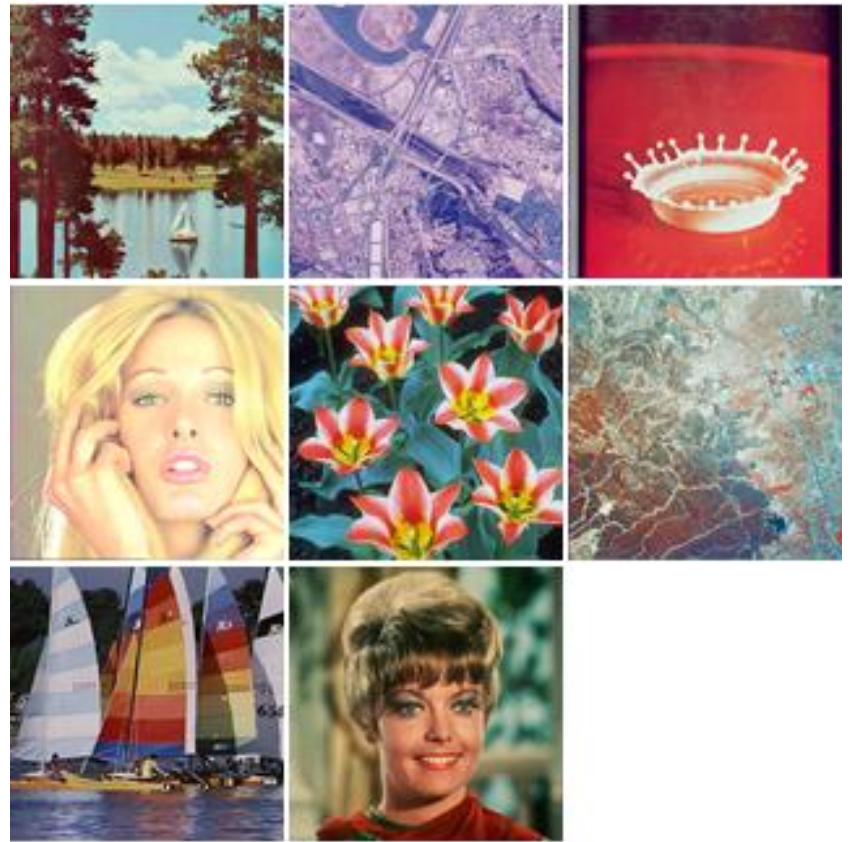

The plain text considered as secret message in the experiments is[14]cut in the length of 31,072 bytes.

For investigating the LSB and IALSB methods, an application is developed to implement the methods and obtain the experimental results. For SLSB method and the related results, [16] is applied.

\section{PERFORMANCE EVALUATION AND RESULTS}

In order to evaluate the quality of the stego images compared to the cover images, following measures are applied[7],[12],[16], [20],[22], and[23]:

Mean Absolute Error $(M A E)=\frac{1}{M N} \sum_{i=1}^{M} \sum_{j=1}^{N}\left|X_{i j}-Y_{i j}\right|(3)$

Mean Square Error $(M S E)=\frac{1}{M N} \sum_{i=1}^{M} \sum_{j=1}^{N}\left(X_{i j}-Y_{i j}\right)^{2}(4)$

$$
L^{p} N o r m=\left(\frac{1}{M N} \sum_{i=1}^{M} \sum_{j=1}^{N}\left(\left|X_{i j}-Y_{i j}\right|\right)^{p}\right)^{1 / p}
$$

$$
\begin{aligned}
& \text { Laplacian Mean Square Error (LMSE) } \\
& =\sum_{i=1}^{M} \sum_{j=1}^{N}\left(L\left(X_{i j}\right)-L\left(Y_{i j}\right)\right)^{2} / \sum_{i=1}^{M} \sum_{j=1}^{N}\left(L\left(X_{i j}\right)\right)^{2}(6)
\end{aligned}
$$

where $L\left(X_{i j}\right)=X_{i+1, j}+X_{i-1, j}+X_{i, j+1}+X_{i, j-1}-4 X_{i j}(7)$

Signal - to - Noise Ratio (SNR)

$$
=\sum_{i=1}^{M} \sum_{j=1}^{N} X_{i j}^{2} / \sum_{i=1}^{M} \sum_{j=1}^{N}\left(X_{i j}-Y_{i j}\right)^{2}(8)
$$

$$
\operatorname{SNR}(d B)=10 \log _{10}(\operatorname{SNR})(9)
$$

Peak Signal - to - Noise Ratio (PSNR)

$$
\begin{gathered}
=M N\left(\max X_{i j}\right)^{2} / \sum_{i=1}^{M} \sum_{j=1}^{N}\left(X_{i j}-Y_{i j}\right)^{2}(10) \\
\operatorname{PSNR}(d B)=10 \log _{10}(P S N R)(11)
\end{gathered}
$$

Normalized Cross Correlation (NCC)

$$
=\sum_{i=1}^{M} \sum_{j=1}^{N} X_{i j} Y_{i j} / \sum_{i=1}^{M} \sum_{j=1}^{N} X_{i j}^{2}
$$

Normalized cross correlation (NCC)

$$
=\sum_{i=1}^{M} \sum_{j=1}^{N} X_{i j} Y_{i j} / \sqrt{\left(\sum_{i=1}^{M} \sum_{j=1}^{N} X_{i j}^{2}\right)\left(\sum_{i=1}^{M} \sum_{j=1}^{N} Y_{i j}^{2}\right)}
$$

where $M$ and $N$ are the number of pixels in the vertical and horizontal dimensions of cover imagerespectively. $X_{i j}$ is the intensity value of a cover pixel whose coordinates are $(i, j)$ in row and column respectively and $Y_{i j}$ refers to the intensity value of corresponding pixel in stego image[7].

Note that the measure (12); NCC from[7],[12], [16], and[22], which is a normalized measure,in some experiments returns some values greater than 1. Due to this, (13) is employed as well from [23] whose results are smaller than or equal to 1. The results of experiments are represented in two tables in order to be categorized based on the number of embedding colors and bits they apply per pixel (payload). Multi-color IALSB may apply three colors and 3, 6, or 9 LSB per pixel which is comparable toLSB method. On the other hand, Singlecolor IALSB which uses one color and 1, 2, 3 LSB per pixel (in the selected color), is comparable to SLSB method.The results are as follows: 


\begin{tabular}{|c|c|c|c|c|c|c|c|c|c|c|}
\hline \multicolumn{2}{|c|}{$\begin{array}{c}\text { Multi-color IALSB } \\
\text { vs. LSB }\end{array}$} & MAE & MSE & L3-Norm & LMSE & $\mathrm{SNR}(\mathrm{dB})$ & $\operatorname{PSNR}(\mathrm{dB})$ & $\begin{array}{l}\mathrm{NCC} \text { from } \\
\text { (12) }\end{array}$ & $\begin{array}{l}\text { NCC from } \\
\text { (13) }\end{array}$ & $\begin{array}{l}\text { Average } \\
\text { bit/pixel }\end{array}$ \\
\hline \multirow{2}{*}{$\frac{2}{n}$} & LSB & 0.4736992 & 0.9464504 & 1.2861368 & 0.001556 & 52.606876 & 57.103834 & 0.9998858 & 0.9999959 & 3 \\
\hline & $\begin{array}{c}\text { Multi-color } \\
\text { IALSB }\end{array}$ & 0.4745405 & 0.7554602 & 1.1176865 & 0.001481 & 53.618729 & 58.115686 & 0.9991522 & 0.9999963 & 1.895 \\
\hline \multirow{2}{*}{$\frac{2}{0}$} & LSB & 0.572603 & 2.486775 & 2.2967699 & 0.0036694 & 48.412885 & 52.909843 & 0.9997619 & 0.9999905 & 6 \\
\hline & $\begin{array}{l}\text { Multi-color } \\
\text { IALSB }\end{array}$ & 0.5308876 & 1.5229263 & 1.7377534 & 0.0029843 & 50.633689 & 55.130647 & 0.9994318 & 0.9999923 & 3.4635 \\
\hline \multirow{2}{*}{$\frac{2}{a}$} & LSB & 0.8214569 & 7.5324951 & 4.2587979 & 0.0114103 & 43.606074 & 48.103032 & 0.9997076 & 0.9999722 & 9 \\
\hline & $\begin{array}{l}\text { Multi-color } \\
\text { IALSB }\end{array}$ & 0.7287823 & 4.1776619 & 3.059052 & 0.008169 & 46.309189 & 50.806147 & 0.9998003 & 0.9999789 & 5.115 \\
\hline
\end{tabular}

Table 1. Performance measures of Multi-color IALSB versus LSB (Average of 20)

\begin{tabular}{|c|c|c|c|c|c|c|c|c|c|c|}
\hline \multicolumn{2}{|c|}{$\begin{array}{c}\text { Single-color IALSB } \\
\text { vs. SLSB }\end{array}$} & MAE & MSE & L3-Norm & LMSE & SNR & PSNR & $\begin{array}{c}\text { NCC from } \\
\text { (12) }\end{array}$ & $\begin{array}{c}\text { NCC from } \\
(13)\end{array}$ & $\begin{array}{l}\text { Average } \\
\text { bit/pixel }\end{array}$ \\
\hline \multirow{2}{*}{$\frac{2}{2}$} & SLSB & 0.4736994 & 0.4737059 & 0.7795451 & 0.0017118 & 55.612718 & 60.109676 & 0.9999049 & 0.9999959 & 1 \\
\hline & $\begin{array}{l}\text { Single-color } \\
\text { IALSB } \\
\end{array}$ & 0.4725033 & 0.4725033 & 0.7788751 & 0.0014428 & 55.623761 & 60.120719 & 0.9994731 & 0.9999961 & 1 \\
\hline \multirow{2}{*}{$\frac{n}{2}$} & SLSB & 0.5238035 & 0.9100225 & 1.2232723 & 0.0032069 & 52.777581 & 57.274539 & 0.9999476 & 0.9999922 & 2 \\
\hline & $\begin{array}{l}\text { Single-color } \\
\text { IALSB }\end{array}$ & 0.5199331 & 0.8962461 & 1.2139305 & 0.0028191 & 52.84385 & 57.340808 & 0.9995667 & 0.9999924 & 2 \\
\hline \multirow{2}{*}{$\frac{2}{n}$} & SLSB & 0.7281796 & 2.5072136 & 2.1831904 & 0.0082947 & 48.376699 & 52.873657 & 0.999879 & 0.9999784 & 3 \\
\hline & $\begin{array}{l}\text { Single-color } \\
\text { IALSB }\end{array}$ & 0.7131233 & 2.3936398 & 2.1301713 & 0.0067849 & 48.580295 & 53.077253 & 0.9997569 & 0.9999794 & 3 \\
\hline
\end{tabular}

Table 2. Performance measures of Single-color IALSB versus SLSB (Average of 20)

The measures (3), (4), (5), and (6)represent the difference and distance between cover image and stego image. Thus, higher value of these measures implies to more distortions of stego image compared to cover image. As shown in the Tables 1 and 2, proposed approaches represent better results supplied by MAE, MSE, L $^{3}$ Norm, and LMSE for all number of embedding bits except the one highlighted by red color. Oppositely, the measures (8), (9), (10), (11), (12), and (13) indicate the similarity between cover image and stego image. Therefore, higher value of these metric implies to less distortion of stego image in comparison to cover image. As represented in the Tables 1 and 2, proposed approaches give better results supplied by SNR, PSNR, and NCCs for all number of embedding bits.

\section{CONCLUSION}

Proposed method presents a new simple type of scatter of secret data over the cover image in two approaches. The secret information can be tunably embedded in the high intensity colors and components of the cover image which cause the modifications be a small proportion of original intensity values and consequently, less distortion of stego image. Multi-color LSB benefits from variable number of embedding component and bit per pixel which makes extraction procedure more undiscoverable to attackers. Two approaches of IALSB specially Single-color IALSB, can be robust against statistical analysis due to modification of only one color of cover pixels and leaving the two others unchanged[12]. Investigating the robustness of proposed method against to steganalyses, applying a steganographic or cryptographic approach to transmit the stego key, and applying a compression method to compress the secret message which leads to less distortion of stego image versus cover image can be considered asfuture works.

\section{REFERENCES}

[1] Rahul Joshi, Lokesh Gagnani, and Salony Pandey, "Image Steganography With LSB," International Journal of Advanced Research in Computer Engineering \& Technology (IJARCET), vol. 2, no. 1, pp. 228-229, January 2013.

[2] Nagham Hamid, Abid Yahya, R. Badlishah Ahmad, and Osamah M. Al-Qershi, "Image Steganography Techniques: An Overview," International Journal of Computer Science and Security (IJCSS), vol. 6, no. 3, pp. 168187, June 2012.

[3] Rahul Joshi, Lokesh Gagnani, and Salony Pandey, "Image Steganography," International Journal of Advanced Research in Computer Engineering \& Technology (IJARCET), vol. 2, no. 1, pp. 224-227, January 2013.

[4] Mehdi Kharrazi, Husrev T. Sencar, and Nasir Memon, "Performance study of common 
image steganography and steganalysis techniques," Journal of Electronic Imaging, vol. 15, no. 4, p. 041104, December 2006.

[5] P. Mohan Kumar and K. L. Shunmuganathan, "Developing a Secure Image Steganographic System Using TPVD Adaptive LSB Matching Revisited Algorithm for Maximizing the Embedding Rate," Information Security Journal, vol. 21, no. 2, pp. 65-70, April 2012.

[6] Abbas Cheddad, Joan Condell, Kevin Curran, and Paul Mc Kevitt, "Digital image steganography: Survey and analysis of current methods," Signal Processing, vol. 90, no. 3, pp. 727-752, March 2010.

[7] Stefan Katzenbeisser and Fabien A. P. Petitcolas, Information Hiding Techniques for Steganography and Digital Watermarking, 1st ed., Stefan Katzenbeisser and Fabien A. P. Petitcolas, Eds. Boston, USA: Artech House, 2000.

[8] V. Lokeswara Reddy, A. Subramanyam, and P. Chenna Reddy, "Implementation of LSB Steganography and its Evaluation for Various File Formats," International Journal of Advanced Networking and Applications (IJANA), vol. 2, no. 5, pp. 868-872, November 2011.

[9] Tayana Morkel, Jan H. P. Eloff, and Martin S. Olivier, "An Overview of Image Steganography," in Proceedings of the ISSA New Knowledge Today Conference, Sandton, South Africa, 2005, pp. 1-11.

[10] Sathiamoorthy Manoharan, "Steganalysis of synthetic low-colour images," in Proceedings of the International Symposium on Information Theory and its Applications (ISITA), Honolulu, Hawaii, USA, 2012, pp. 784-788.

[11] Kaustubh Choudhary, "Properties of images in LSB plane," IOSR Journal of Computer Engineering (IOSRJCE), vol. 3, no. 5, pp. 816, August 2012.

[12] Juan J. Roque and Jesus M. Minguet, "SLSB: Improving the Steganographic Algorithm LSB," in Proceedings of the 7th International Workshop on Security in Information Systems (WOSIS), Milan, Italy, 2009, pp. 1-11.

[13] Sorina Dumitrescu, Xiaolin $\mathrm{Wu}$, and Zhe Wang, "Detection of LSB steganography via sample pair analysis," IEEE Transactions on Signal Processing, vol. 51, no. 7, pp. 19952007, July 2003.
[14] Andrew D. Ker, "Improved detection of LSB steganography in grayscale images," in Proceedings of 6th International Workshop of Information Hiding, Toronto, Canada, 2004, pp. 97-115.

[15] Juan J. Roque. (2010, October) Steganography Studio 1.0.2. Software (Executable Jar File). [Online]. http://sourceforge.net/projects/stegstudio/files

[16] Juan J. Roque. (2010, October) Steganography Studio. [Online]. http://stegstudio.sourceforge.net/bibliography .html

[17] Marten Van Dijk and Frans Willems, "Embedding information in grayscale images," in Proceedings of the 22nd Symposium on Information and Communication Theory in the Benelux, Enschede, Netherlands, 2001, pp. 147-154.

[18] Miroslav Goljan, Jessica Fridrich, and Taras Holotyak, "New blind steganalysis and its implications," in Proceedings of the SPIE, Electronic Imaging, Security, Steganography, and Watermarking of Multimedia Contents VIII, San Jose, CA, USA, 2006, pp. 1-13.

[19] Saeed Mahmoudpour and Sattar Mirzakuchaki, "Hardware architecture for a message hiding algorithm with novel randomizers," International Journal of Computer Applications (IJCA), vol. 37, no. 7, pp. 46-53, January 2012.

[20] Weiqi Luo, Fangjun Huang, and Jiwu Huang, "Edge adaptive image steganography based on LSB matching revisited," IEEE Transactions on Information Forensics and Security, vol. 5, no. 2, pp. 201-214, June 2010.

[21] Ahmet M. Eskicioglu and Paul S. Fisher, "Image quality measures and their performance," IEEE Transactions on Communications, vol. 43 , no. 12 , pp. 2959 2965, December 1995.

[22] Yong Seok Heo, Kyoung Mu Lee, and Sang Uk Lee, "Robust stereo matching using adaptive normalized cross-correlation," IEEE Transactions on Pattern Analysis and Machine Intelligence, vol. 33, no. 4, pp. 807822, April 2011. 